\title{
Establishment of Signaling Interactions with Cellular Resolution for Every Cell Cycle of Embryogenesis
}

\author{
Long Chen, ${ }^{*, 1}$ Vincy Wing Sze Ho, ${ }^{\dagger, 1}$ Ming-Kin Wong, ${ }^{\dagger, 1}$ Xiaotai Huang, ${ }^{\ddagger, 1}$ Lu-yan Chan, ${ }^{+}$Hon Chun Kaoru Ng, ${ }^{\dagger}$ \\ Xiaoliang Ren, ${ }^{\dagger}$ Hong Yan,* and Zhongying Zhao ${ }^{\dagger, \$, 2}$ \\ *Department of Electronic Engineering, City University of Hong Kong, China, 'Department of Biology, Hong Kong Baptist \\ University, China, ${ }^{\ddagger}$ School of Computer Science and Technology, Xidian University, Xi'an, 710126 China, and §State Key \\ Laboratory of Environmental and Biological Analysis, Hong Kong Baptist University, China \\ ORCID IDs: 0000-0002-4088-3819 (M.-K.W.); 0000-0003-2743-9008 (Z.Z.)
}

\begin{abstract}
Intercellular signaling interactions play a key role in breaking fate symmetry during animal development. Identification of signaling interactions at cellular resolution is technically challenging, especially in a developing embryo. Here, we develop a platform that allows automated inference and validation of signaling interactions for every cell cycle of Caenorhabditis elegans embryogenesis. This is achieved by the generation of a systems-level cell contact map, which consists of 1114 highly confident intercellular contacts, by modeling analysis and is validated through cell membrane labeling coupled with cell lineage analysis. We apply the map to identify cell pairs between which a Notch signaling interaction takes place. By generating expression patterns for two ligands and two receptors of the Notch signaling pathway with cellular resolution using the automated expression profiling technique, we are able to refine existing and identify novel Notch interactions during C. elegans embryogenesis. Targeted cell ablation followed by cell lineage analysis demonstrates the roles of signaling interactions during cell division in breaking fate symmetry. Finally, we describe the development of a website that allows online access to the cell-cell contact map for mapping of other signaling interactions by the community. The platform can be adapted to establish cellular interactions from any other signaling pathway.
\end{abstract}

KEYWORDS Cell contact; Notch signaling; cell lineage; C. elegans; division asymmetry

YMMETRY breaking in cell division timing and cell fate specification has long been a focus of developmental biology. Intercellular signaling plays a key role in breaking these symmetries (Yochem et al. 1988; Clevers and Nusse 2012; Sawa 2012; Greenwald 2013; Zacharias et al. 2015), although maternal control is critical for establishing polarity during early development (Rose and Gonczy 2014). For example, a Notch signaling interaction is necessary for fate asymmetry between cells ABa and ABp (Mickey et al. 1996; Priess 2005), whereas a Wnt interaction is required for both fate asymmetry and division asynchrony between cells

Copyright @ 2018 by the Genetics Society of America

doi: https://doi.org/10.1534/genetics.118.300820

Manuscript received February 13, 2018; accepted for publication March 19, 2018; published Early Online March 22, 2018.

Supplemental material available at Figshare: https://doi.org/10.25386/genetics. 6007709.

${ }^{1}$ These authors contributed equally to this work.

${ }^{2}$ Corresponding author: Department of Biology, State Key Laboratory of Environmental and Biological Analysis, Hong Kong Baptist University, 224 Waterloo Road, Kowloon Tong, Hong Kong, China. E-mail: zyzhao@hkbu.edu.hk
EMS and P2 in a four-cell Caenorhabditis elegans embryo (Rocheleau et al. 1997). The Notch interaction is achieved by a contact between the P2 cell, which expresses a Notch ligand called apx-1, and the $\mathrm{ABp}$ but not the $\mathrm{ABa}$ cell, although both the latter cells express the Notch receptor $g l p-1$ (Mickey et al. 1996). This demonstrates that a contact between cells is essential for triggering a signaling interaction to drive differential fate specification (Good et al. 2004). A similar scenario is observed for the Wnt interaction between the EMS and P2 cells, which is necessary for asymmetric division of the former into MS and E cells during C. elegans embryogenesis (Goldstein 1992; Rocheleau et al. 1997). Notably, the two pathways are used repeatedly throughout development in a cellular context-dependent fashion to establish further asymmetries in fate specification or division timing (Huang et al. 2007; Zacharias et al. 2015). For example, in a 12-cell C. elegans embryo, the four great-granddaughters of $A B$ express the Notch receptor GLP-1, but only two of them, i.e., ABalp and ABara, are in contact with a Notch ligandexpressing cell, MS, leading to their differential differentiation 
into mesodermal and ectodermal fates, respectively (Hutter and Schnabel 1994; Shelton and Bowerman 1996). Importantly, signaling interactions from the same pathway may have an opposite consequence depending on their timing or cellular context. For example, the first Notch interaction inactivates its targets, tbx-37/38 (Good et al. 2004); whereas the second one activates its targets including PHA-4, a FoxA transcription factor required for pharynx organogenesis (Priess 2005). These time-dependent signaling events indicate that dissecting signaling interactions with precise spatial and temporal resolution would be essential for a thorough understanding of symmetry breaking during metazoan development.

One of the biggest challenges in defining a signaling interaction during embryogenesis is the establishment of cell identity, especially in an embryo with a large number of cells (Keller et al. 2008; Zacharias and Murray 2016). Another challenge is that one must have access to the cellular expression patterns of signaling molecules for each cell cycle. These requirements inhibit the functional characterization of cellular signaling during rapid development. This is because defining a signaling interaction requires knowledge of the identities of cell pairs that are in contact with each other, with one expressing a ligand and the other a receptor.

The development of cell-tracking techniques using timelapse three-dimensional (3D) [hereafter referred to as fourdimensional (4D)] microscopy has greatly facilitated cell lineage analysis (Schnabel et al. 1997, 2006, Zhao et al. 2008, 2010a; Muzzey and van Oudenaarden 2009). In particular, a recently developed automated lineaging technique allows routine tracing of cell division and single-cell expression profiling in a $C$. elegans embryo with up to 350 cells within approximately half an hour, and up to the last round of cell division of embryogenesis in $\sim 1$ day (Bao et al. 2006; Murray et al. 2008; Richards et al. 2013; Du et al. 2014; Shah et al. 2017). This technique makes it possible to infer signaling interactions at cellular resolution for every cell cycle (Figure 1) because the output of automated lineaging contains quantitative positional information for nuclei of all cells for every minute during embryogenesis, thus allowing systematic modeling of cell contacts with exceptional spatial and temporal resolution. A cell contact map up to the $\sim 150$-cell stage was previously reported for the C. elegans embryo purely based on Voronoi modeling (Hench et al. 2009). However, the map suffers from several caveats. First, it was generated using a single "composite" embryo assembled from six different embryos, each of which was partially resolved for cell lineage. Given the variability in embryo size, shape, and developmental timing (Hara and Kimura 2009; Greenan et al. 2010; Moore et al. 2013; Ho et al. 2015), it would be problematic to superimpose the six embryos into a single embryo for modeling of cell contacts. Second, a thorough validation of the modeling results was not performed. Many cell contacts that are brief in duration and/or have a minimal contact area may not be consequential. As a result, a relatively high false-positive rate is unavoidable if these issues are not taken into account. Finally, the map covers only the $\sim 150$-cell stage, but a $C$. elegans embryo does not hatch until it develops into 558 cells (Sulston et al. 1983). Therefore, a more reliable cell contact map that covers cells born at a later stage of embryogenesis is necessary for the dissection of cell signaling. Here, we present a platform that allows the automated inference of cellular signaling for every cell cycle up to the $\sim 350$-cell C. elegans embryo. Applying the platform to the Notch signaling pathway demonstrated consecutive signaling events over cell cycles for the breaking of cell fate symmetry.

\section{Materials and Methods}

\section{Modeling of cell-cell contact}

Prediction of cell surface is performed using the Voronoi segmentation algorithm (Aurenhammer 1991; Okabe et al. 2000) with the "Voro++" library (Rycroft 2009) using the output from StarryNite as an input, which contains 3D coordinates for all nuclei at a 1.5-min interval from 4 to 350 cells of a C. elegans embryo. One caveat of the method is the segmentation of the cells located at the edge of an embryo, where a falsepositive cell contact may be predicted, as reported previously (Hench et al. 2009). To solve this issue, for each embryo, a 3D convex hull was generated as a proxy for the embryo boundary. Given the reproducible migration of cells at the embryo boundary, the cell surface and contact areas were computed with cells' coordinates and the 3D convex hull with Voro ++ .

3D coordinates from 91 wild-type $C$. elegans embryos were individually modeled to define cell contacts for each time point $(1.5 \mathrm{~min}$ ) for all embryos. To evaluate the variability of cell contacts among embryos, cell contact areas were compared against each embryo using cell stage, i.e., the number of cells in a given embryo, rather than the absolute developmental time. This would minimize the complications associated with variability in developmental timing.

\section{Visualization of cell boundary at the desired stage}

A strain, ZZY0535, was made by crossing the lineaging strain RW10029 expressing GFP lineaging markers with strain OD84 expressing a membrane marker, Ppie-1::mCherry::PH (PLC181) (see Supplemental Material, Table S3). The three markers were rendered triply homozygous.

For visualization of cell contacts by fluorescence membrane labeling, a four-cell embryo with desired developmental timing was selected for 4D imaging with a Leica SP5 confocal microscope using similar settings as those used for automated lineaging, until the embryo developed to the desired stage. Timing for the presence of a cell of interest was estimated based on our lineaging results of the 91 wild-type embryos (Ho et al. 2015). Imaging with live data mode was switched to normal mode to take a single stack consisting of 110 focal planes with suitable AOTF compensation using a pinhole of 1.6 arbitrary unit (AU) and three-line accumulation . Images were acquired from both GFP and mCherry channels. The identity of the cell of interest was resolved by manually navigating through the image stacks using Leica Application Suite X 

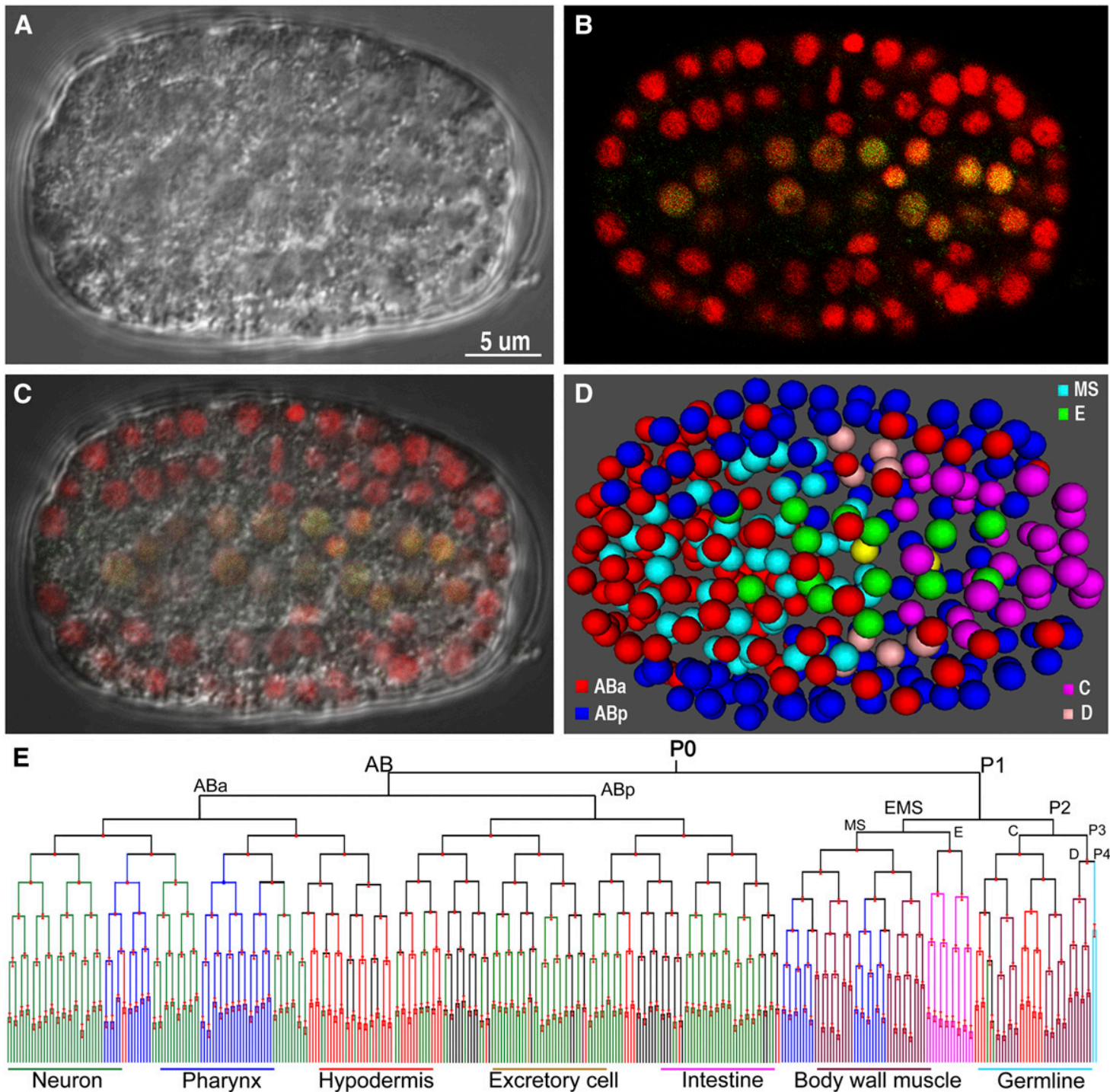

Figure 1 Overview of a 350-cell stage C. elegans embryo. (A) Nomarski micrograph of a C. elegans embryo of approximately the 350-cell stage. (B) Epifluorescence micrograph showing superimposed nuclear expression patterns of lineaging markers (red) and a pharynx marker, PHA-4 (green). (C) Superimposed micrograph from (B) and (C). (D) Three-dimensional space-filling model of a 350-cell embryo. Cells are differentially color-coded based on their lineal origins. MS, cyan; E, green; Aba, red; $A B p$, blue; $C$, magenta; and D, peach. (E) Cell lineage tree up to the 350-cell stage. Cell lineages are differentially colored based on cell fate except the undifferentiated cell fates that are colored in black.

(LAS X). The 3D stack of the embryo was used to reconstruct the 3D volume projection with LAS X. The embryo was rotated to a proper orientation to facilitate visualization of the desired cell boundary. Part of the embryo was cut open across different axes for visualization of contacts.

\section{Cell ablation coupled with cell lineage analysis}

For cell ablation coupled with automated lineaging, a four-cell embryo with desired orientation was selected for 4D imaging until the cell targeted for ablation was born. Manual tracing of the targeting cell was performed with the help of the lineaging markers. Immediately after the target cell completed mitosis, imaging was terminated and the following procedures were performed within 1.5 min: switching of the imaging mode from live data mode to normal mode; focusing on the middle plane of the target cell nucleus by fine-tuning the Z-Galvo; selecting the bleaching point from the panel and creating a region of interest in the middle of the target cell nucleus in a preview panel; turning off all the fluorescence detectors except the one for the DIC and switching the filter to "substrate"; setting the bleaching time (40 sec for ABala, $20 \mathrm{sec}$ for all others); temporally closing the shutters for all the wavelengths except the pulsed diode laser (800-B; PicoQuant), which emits a $405-\mathrm{nm}$ laser beam; tuning it to $100 \%$ intensity and starting the bleaching; and, once completed, switching back to the live data mode and resuming the 4D imaging as usual.

\section{Generation of single-copy transgenic lines}

A single-copy transgene consisting of a fusion between the promoter of a Notch ligand/receptor and GFP was generated 
using the miniMos technique (Frøkjær-Jensen et al. 2014). The primer sequences used to amplify the promoter sequences are listed in Table S4. The miniMos targeting vector pCFJ909 was modified to include a genomic coding region of his-24 upstream of the GFP coding sequence to facilitate nuclear localization for automated lineal expression profiling, as previously described (Zhao et al. 2010b). Multiple independent strains were produced for each promoter. A single strain that shows expression patterns consistent with the remaining ones was genotyped by inverse PCR and crossed with the lineaging strain RW10226. Both lineaging and Notch markers were rendered homozygous for automated lineaging and lineal expression profiling, as previously described (Zhao et al. 2010b).

\section{D live cell imaging, automated lineaging, and profiling of lineal gene expression}

Imaging was performed in a similar way to that described previously (Shao et al. 2013). Briefly, lineaging strain RW10226 (Zhao et al. 2010a), ubiquitously expressing nuclear mCherry, was crossed with the strain expressing a fusion between the promoter of a Notch component and GFP. Both the lineaging markers and the promoter fusion were rendered homozygous before lineaging. 4D imaging stacks (roughly $0.7 \mu \mathrm{m} /$ stack) were sequentially collected for both GFP and red fluorescent protein (mCherry) channels at a 1.5-min interval for a total of 240 time points using a Leica SP5 confocal microscope, as previously described (Shao et al. 2013). Automated profiling of lineal expression was performed, as previously described (Zhao et al. 2010b).

\section{Worm strains and maintenance}

All the animals were maintained on NGM plates seeded with OP50 at room temperature unless stated otherwise. The genotypes of the strains used in this paper are listed in Table S3.

\section{Data availability}

Strains are available upon request. The authors affirm that all data necessary for confirming the conclusions of the article are present within the article, figures, and tables. Cell-cell contacts can also be accessible through the following link: http:// ccccm.bionetworks.ml/. Supplemental material available at Figshare: https://doi.org/10.25386/genetics.6007709.

\section{Results}

A time-lapse cell-contact map from 4- to 350-cell C. elegans embryos

To facilitate the precise assignment of cell pairs between which a potentially functional signaling interaction takes place, we performed modeling analysis of cell-cell contact over the proliferative stage of $C$. elegans embryogenesis from 4 to 350 cells. Specifically, 4D coordinates from 91 wild-type embryos generated previously by automated lineaging (Ho et al. 2015) were individually used as an input for the Voronoi algorithm to model cell surfaces, from which the contacting area is computed between a cell pair (see Materials and Methods).
Instead of using partial 4D coordinates from different embryos, as in a previous study (Hench et al. 2009), the 91 coordinate sets used here were each derived from single intact embryos, which minimizes the issues associated with normalization steps for cell size, embryo shape, and developmental timing.

It is conceivable that many cell contacts may not be relevant to cell signaling, either due to their short duration or small contact area. To increase the modeling accuracy, we adopted the following criteria to define an effective cell contact, which is referred to as cell contact hereafter for simplicity unless stated otherwise. First, a contact area is required to be at least $6.5 \%$ of the average cell surface areas of all cells present at the same time point (Figure 2A). Second, this criterion must be satisfied for at least two consecutive time points $(\sim 1.5 \mathrm{~min}$ per time point) (Figure 2C, see details below). Third, these two criteria must be reproducible in $\geq 95 \%$ of the 91 wildtype embryos (i.e., in 87 of 91 embryos; Figure 2B). As a result, we predicted a total of 1114 cell contacts from the 4- to 350-cell stage (Table S1). The predicted contact areas were highly reproducible among the 91 embryos with a Pearson correlation coefficient $(r)$ of at least 0.8 between any two independent embryos (Figure 2D). The predicted cell contact can be readily validated via ubiquitous and simultaneous labeling of cell membranes and nuclei with resolved cell identities (Figure 2E).

We adopted the criterion of a $6.5 \%$ contact area based on the well-established second Notch interactions in the C. elegans embryo (Mickey et al. 1996). This interaction occurs between a Notch ligand-expressing cell (MS) and two Notch receptorexpressing cells (ABalp and ABara) in a 12-cell embryo, but not in their sisters (ABala and ABarp), which leads to specification of their pharyngeal fate. We first individually computed the contact areas between MS and each of the four $A B$ descendants for the 91 wild-type embryos. Given the variability in contact area between the embryos, we next plotted the occurrence of the four contacts (any contact with a contacting area $>0$ ) in the 91 embryos against the ratio of actual contact area relative to average cell surface areas of all cells present at the current time point. Occurrence distributions of both individual (Figure S1) and aggregated (Figure 2A) plots demonstrated a normal distribution. We observed a clear demarcation between cell pairs with (between MS and ABala or ABarp) and without (between MS and ABalp or ABara) a functional contact at a ratio of $\sim 6.5 \%$ of the actual contact area relative to the average cell surface area of all cells at the current time point (Figure 2A). Therefore, we used the ratio of $6.5 \%$ as a cutoff for defining an effective contact. Variability in actual cell contact was observed not only between MS and the four $\mathrm{AB}$ descendants, but also in other cells from 4 to 350 cells in the 91 embryos (Figure 2B). Therefore, we required that if a contact is reproducibly observed in $95 \%$ of all the 91 embryos, it could be defined as an effective contact. To further reduce our false-positive rate in calling an effective cell contact, we required a contact that lasts for at least two consecutive time points $(\sim 3 \mathrm{~min})$. We set this filter because 

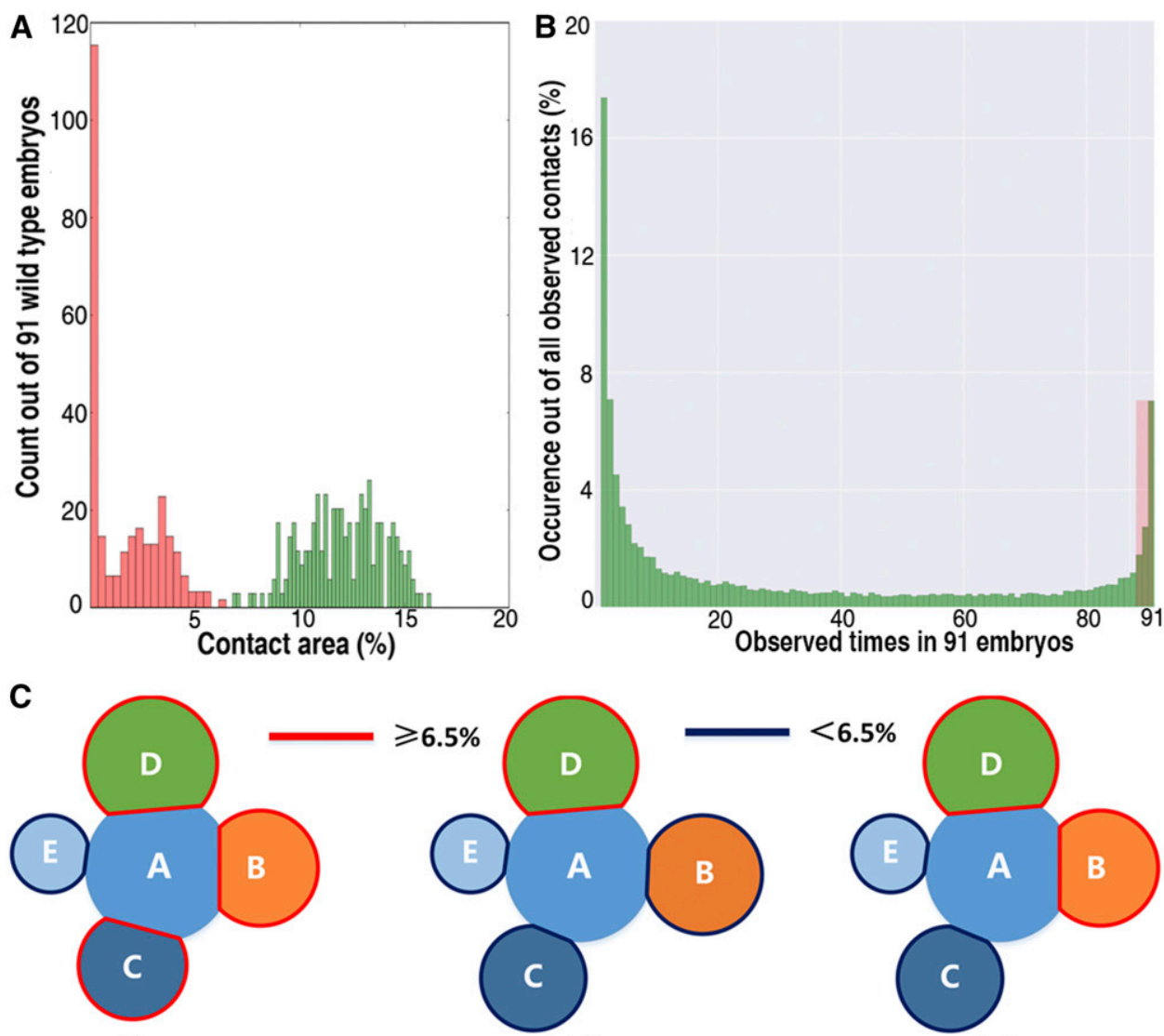

T

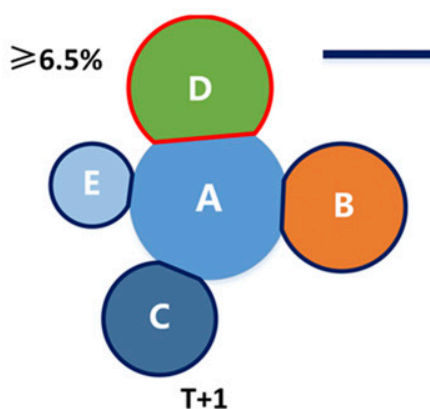

E

D
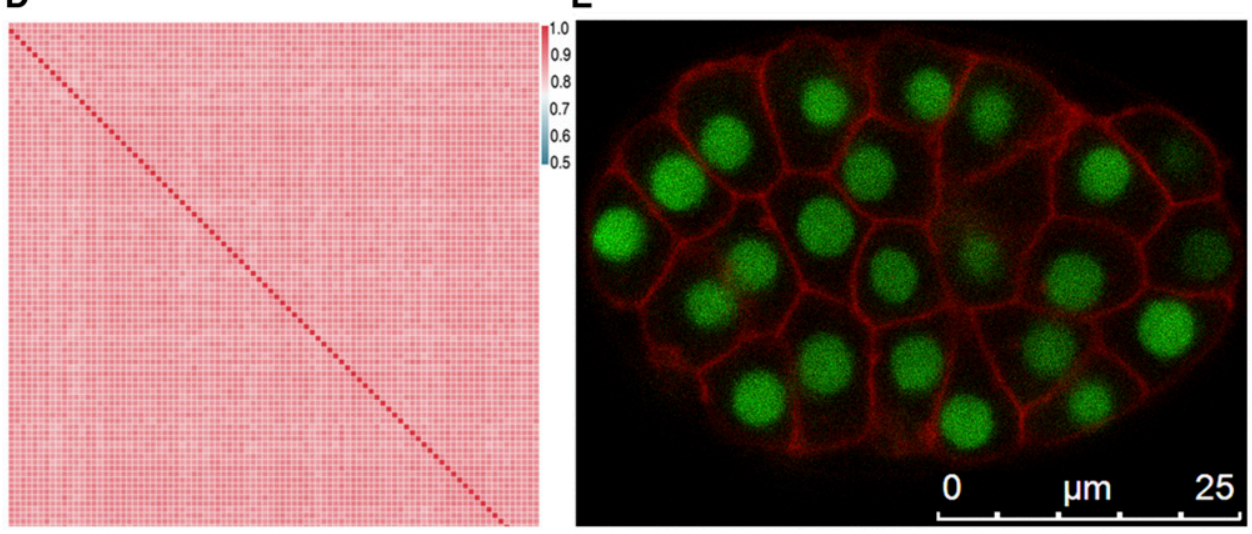

Figure 2 Modeling of cell-cell contact during $C$. elegans embryogenesis. (A) Demarcation of the ratio of contact areas between cells with and without a functional contact. Shown is the occurrence distribution of modeled contact areas between cell pairs that are known to have (green) or not to have (brown) the second Notch (see main text) interactions in 91 embryos. Percentage of contact area out of average cell surface area of all cells in the current time point is plotted on the $x$-axis and the number of embryos with a given ratio out of 91 wild-types on $y$-axis. (B) Distribution of any contacts (contact area $>0$ ) in 91 wild-type embryos. The $y$-axis denotes the percentage of a given contact out of all observed contacts and the $x$ axis the observed times for a given contact out of 91 embryos. Contacts with > 95\% reproducibility (i.e., observed in 87 out of 91 embryos) are shaded in red. (C) A diagram showing the definition of effective cell contact with cell $A$ over three consecutive time points. A contact area that is bigger or smaller than $6.5 \%$ of the average surface areas of all the cells is differentially colored in red and blue lines, respectively. For a given cell pair, only a cell contact area that is $>6.5 \%$ for at least two consecutive time points ( $\sim 3 \mathrm{~min}$ ) is defined as an effective cell contact. (D) Heat map of mutual Pearson correlation's coefficient (r) of contact areas for all cells between 91 individual wild-type embryos. Both the $x$ - and $y$-axes denote the coefficient of an individual embryo against another. (E) An example of a 40-cell C. elegans embryo expressing GFP in nuclei and membrane marker $\mathrm{PH}(\mathrm{PLC} 1 \delta 1)$ in the cell membrane (red). our temporal resolution is $1.5 \mathrm{~min}$, meaning that the duration of any contact shorter than this will be assigned as $1.5 \mathrm{~min}$. This temporal requirement ensures that an effective cell contact lasts for $\geq 1.5 \mathrm{~min}$.

A previous study suggested the substantial effect of pressure applied to an embryo during imaging on the prediction of cell-cell contact (Hench et al. 2009). We tested the effect of such pressure by examining whether the hatching rates are similar between pressured (mounted) and unpressurized (unmounted) embryos (those laid freely on an NGM plate). If the hatching rates are comparable, after the hatched larvae grow up, their brood sizes would be comparable. We found that all mounted and unmounted embryos (25 in each con- dition) hatched, and that the brood sizes were also comparable between the mounted and unmounted embryos (Figure S2), suggesting that pressure applied on the embryos for mounting was unlikely to affect the important cell contacts during C. elegans embryogenesis.

\section{Comparison of performance between our and a previous contact map}

A previous cell contact map was generated with a modeling algorithm similar to that used here but using a single composite embryo assembled from six different embryos (Hench et al. 2009). The cell lineage for each embryo was partially resolved owing to the difficulty in establishing cell identities 
Table 1 Comparison of our cell contact map with a previous map (Hench et al. 2009) and the contacting cell pairs with known Notch signaling interaction

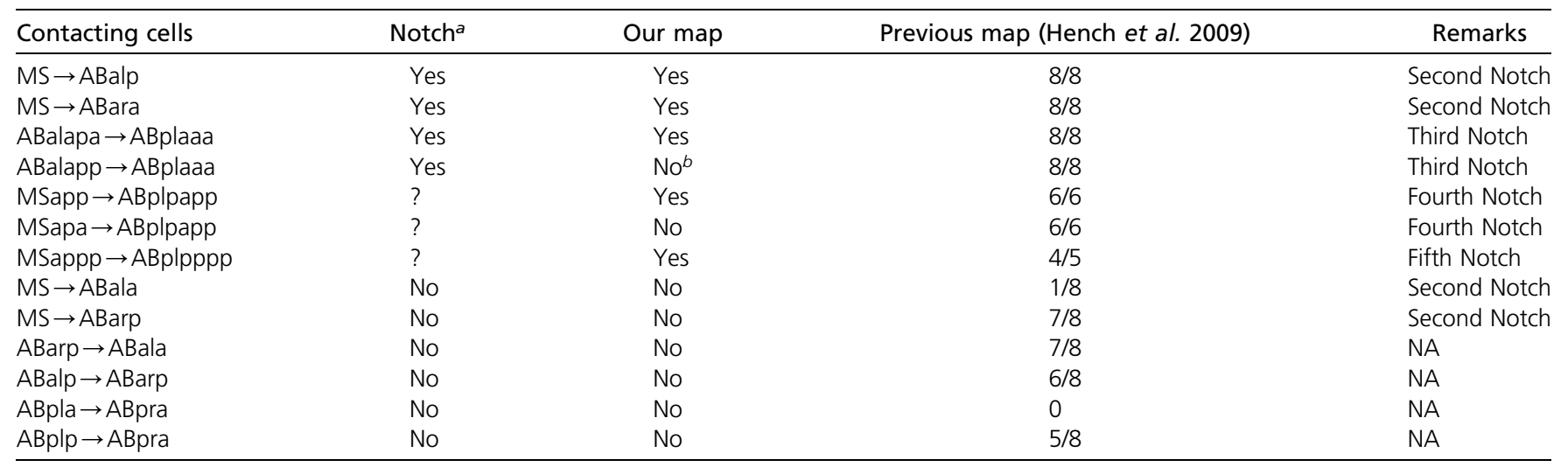

? indicates ambiguity in signaling cell identity. NA, not applicable.

${ }^{a}$ Existing knowledge on Notch signaling.

${ }^{b}$ Could be a false negative due to cutoff of contact area.

on both sides of an embryo. Notably, cell contact was defined mainly based on whether there was any physical contact regardless of the size and duration of a contact, making it prone to a relatively high false-positive rate. The spatial and temporal constrains that we used for modeling are expected to reduce the rate of false positives.

To compare the performance between our and the previous contact maps, we contrasted a subset of cell contacts relevant to well-established Notch signaling interactions (Table 1). It was expected that our modeling contacts would agree well with the contacts based on the second Notch interactions because they were used as a training set for our contact modeling. Notably, nearly one-half of the cell contacts predicted previously were false positives when compared with the experimentally verified ones, whereas our predictions agreed well with the experimental data from multiple Notch interactions (Table 1), indicating that our modeling method substantially outperforms the previous method in terms of accuracy.

\section{Lineal expression of Notch receptors and ligands derived from a single-copy transgene}

Knowledge of the time-lapse expression of a ligand and its receptor in a signaling pathway at the cellular level with high temporal resolution is critical for assigning a cell pair between which a signaling interaction takes place. However, such knowledge is either absent or present at poor spatiotemporal resolution, especially during the proliferative stage of embryogenesis, thus preventing effective assignment of a signaling interaction. For example, existing expression patterns on Notch pathway components in C. elegans were obtained through either a transgenic study, antibody staining, or their combination (Mello et al. 1994; Mickey et al. 1996; Moskowitz and Rothman 1996). Most of the transgenic assays are based on extrachromosomal arrays (Moskowitz and Rothman 1996) or biolistic bombardment (Murray et al. 2012). The expression patterns generated from these transgenic strains may suffer from increased perdurance of fluorescent reporters by extra copies of transgenes or uncertainty in regulatory sequences incorporated into host cells.

To generate the embryonic expression pattern of a Notch component that more likely mimics its native expression at cellular resolution for each cell cycle, we first produced multiple independent transgenic strains carrying a single copy of a fusion between GFP and a promoter sequence from a Notch component using the miniMos technique (FrøkjærJensen et al. 2014), including two functionally redundant receptors, lin-12 and glp-1, and two ligands, apx-1 and lag2. A single strain that showed consistent expression with at least one other transgenic copy was used to map the reporter's lineal expression using automated lineaging and expression profiling technology (Murray et al. 2008). glp-1 shows specific expression in the descendants of ABarpap and ABplaaa (Figure 3, A, B, G, and J). These will generate hypodermal cells found in the head (Sulston et al. 1983). Dim expression was also observed in the descendants of MSaa and MSpa (Figure S2, A and F). Notably, our expression patterns are roughly comparable with those derived from transgenic strains generated with biolistic bombardment in $\mathrm{AB}$ (Murray et al. 2012), but expression was observed in more cells in the bombardment strains. Because the promoter sequences are similar in size, it remains likely that the expression conferred by the single-copy transgene may be too dim to be seen. Expression of the other Notch receptor, lin-12, is mainly observed in the descendants of ABplp, ABprp, and ABplaaa (Figure 3, C, D, H, and J). No expression was observed in the P1 sublineage (Figure S3, B and G). One Notch ligand, apx-1, showed expression mainly in the descendants of ABala, ABpl(r)apaa (Figure 3, E, F, I, and J), MSppapp, and MSppppp (Figure S3, C and H). We did not observe the expression of lag-2 in the ABalap descendants, as reported previously (Moskowitz and Rothman 1996). A complete list of cells expressing Notch ligands and receptors is shown in Table S2. When combined with the cell contact map, the 


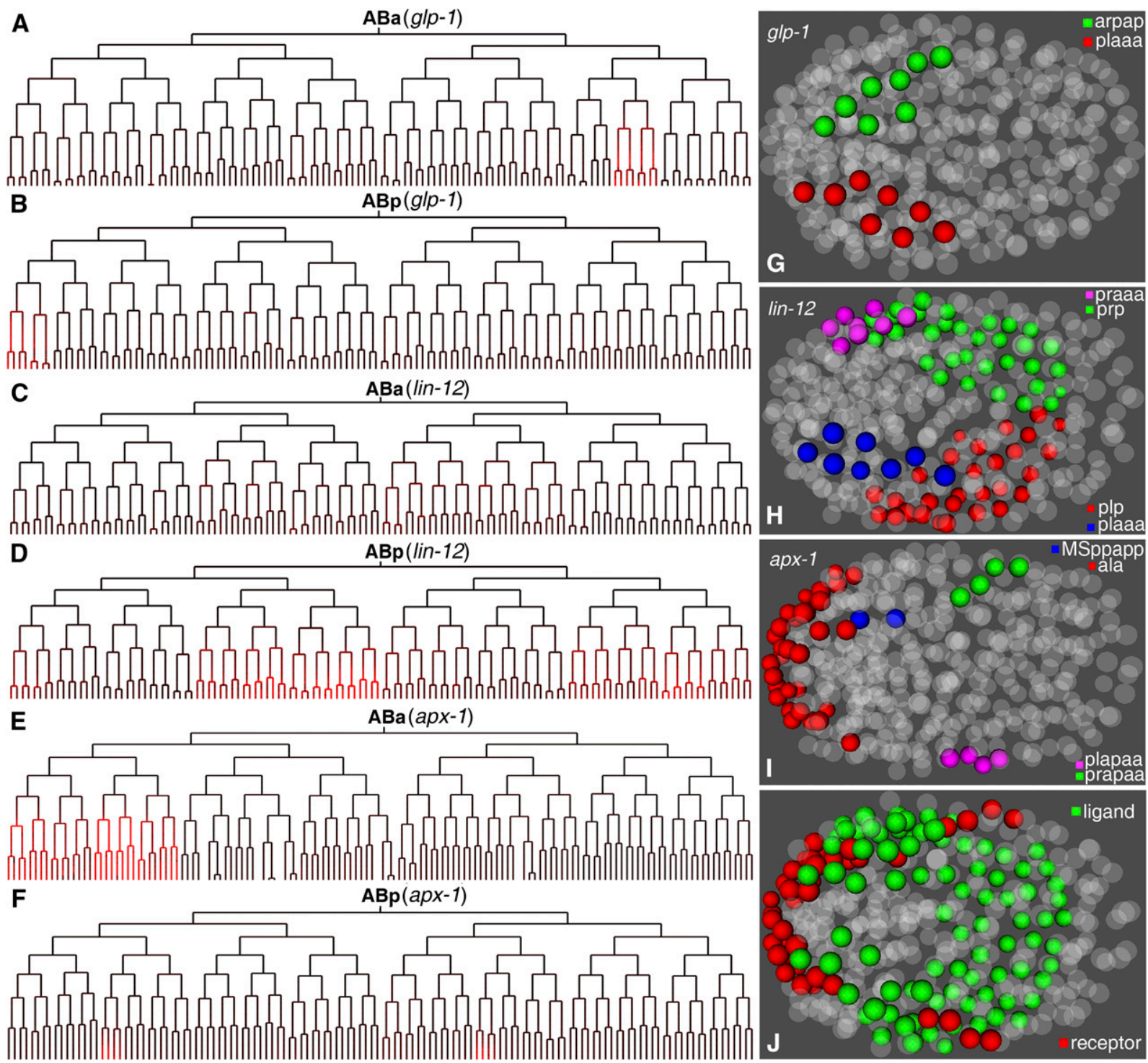

Figure 3 Expression of Notch ligands and receptors in a C. elegans embryo. (A-F) Lineal expression (red) of two Notch receptors, g/p-1and lin-12, and one ligand, apx-1, in the ABa and ABp lineage up to the 350-cell stage. (G-I) Spatial expression of the three genes differentially color-coded based on their lineal origins. (J) Combined spatial expression patterns of the three genes between the ligand, apx-1 (red), and the two receptors, $g / p-1$ and lin-12 (green).

lineal expression of these Notch components at a 1.5 -min interval over development will not only allow the validation of existing Notch signaling interactions, especially at a stage with tens to hundreds of cells, but also holds promise for the identification of novel cell pairs between which a signaling interaction may take place. We illustrate the applications in detail below.

\section{Refinement of the proposed cell pairs for third Notch interaction in C. elegans embryos}

The third Notch signaling between signaling cell ABalapp and signal-receiving cell ABplaaa was proposed mainly based on the expression timing of a Notch ligand, lag-2, in ABalapp and a Notch receptor, lin-12, in ABplaaa (Moskowitz and Rothman 1996). To confirm the signaling interaction and examine its functional redundancy, we took advantage of our timelapse cellular expression patterns of both Notch receptors and two different ligands and aligned them against our modeled cell contacts. If a cell contact is observed between two cells with one expressing a ligand and the other a receptor, it is plausible that a signaling interaction takes place between the two. In addition to lag-2 (Moskowitz and Rothman 1996), we observed the expression of another Notch ligand, $a p x-1$, in the descendants of ABala (Figure 3E). Our reporter assay revealed that both Notch receptors, lin-12 and glp-1, are expressed in the left-head precursor ABplaaa (Figure 3, B and D). Despite the expression of apx-1 in all of the descendants of ABala (Figure 3E), only one of the ABala daughters, ABalap, had cell contact with the left-head precursor ABplaa based on our modeling results (Table S1), suggesting a specific 
signaling interaction between the two, which is consistent with previous cell-ablation results (Hutter and Schnabel 1995; Moskowitz and Rothman 1996). Notably, expression of the Notch ligand lag- 2 and the Notch receptor lin-12 by LacZ-based transgenic assay suggested the signaling interaction at a later stage (i.e., between ABalapp and ABplaaa) (Moskowitz and Rothman 1996). However, our cell contact data suggest that $\mathrm{ABalapa}$ may play a bigger role than $\mathrm{ABa}-$ lapp in signaling the left-head precursor (Figure 4). The three cells stay in different $\mathrm{z}$ planes (Figure 4, A-C). Both daughters of ABalap express apx-1, but the relative contact area with ABplaaa is much greater for ABalapa (16.6\%) than for ABalapp (5\%) (Table S1). In addition, the daughters of ABalapa, but not those of ABalapp, are in contact with those of the daughters of the left-head precursor (Movie S1), which further supports the more important role of ABalapa in signaling ABplaaa than ABalapp. These results suggest that the signaling effect in cell fate specification is achieved through consecutive signaling in multiple generations. It remains possible that two cells signal ABplaaa redundantly. Our reporter assay also showed that both Notch receptors may be redundantly involved in the signaling event, refining the previous finding that only a single ligand and receptor are involved in the third signaling event (Moskowitz and Rothman 1996).

\section{Functional validation of the proposed cell pairs for the third Notch interaction}

To experimentally validate the third Notch interaction, we first used cell membrane labeling coupled with cell lineage analysis (see Materials and Methods). Specifically, we performed 4D live cell imaging of a C. elegans embryo ubiquitously expressing a nuclear and a membrane marker from the four-cell stage up to the desired stage, as estimated by wildtype lineaging trees (Ho et al. 2015). We then took a single 3D stack consisting of 110 focal planes for both GFP (nuclear) and mCherry (membrane) channels, which were rendered as a 3D projection (Figure 4D). The 4D images allowed manual or automated tracing of cell identities, whereas the 3D projection permitted the establishment of cell boundaries (Figure 4, A-C). In agreement with our modeling results, the cell membrane labeling showed a higher confidence of contact with the left-head precursor by cell ABalapa than by cell ABplapp (Figure 4, D and E). The contact seems not obvious in modeled cell boundaries (Figure 4F). This may be mainly due to the positional differences across the $z$-axis.

We next verified whether the predicted signaling cell functions as expected using the cell-ablation technique. Given that apx-1 is expressed in all ABala descendants, we decided to test whether the signaling interaction takes place in multiple generations, as stated above, by a combination of cell ablation and Notch target expression. We first ablated the cell ABala and examined the expression of a Notch target, ref-1, which is known to be expressed in the precursors of both the left and right heads (Neves and Priess 2005; Murray et al. 2012) (Figure 4, G and H and Figure S4A). As expected, ablation of the cell led to the specific loss of ref- 1 expression in the left-head precursor (Figure $4 \mathrm{H}$ and Figure S4A), demonstrating that the ligands expressed in this cell or its daughters are responsible for the signaling interaction. We next ablated the posterior daughter of ABala, ABalap, and reexamined the expression of ref-1. Interestingly, ablation of the cell abolished the ref- 1 expression in the posterior but not in the anterior descendants of ABplaaa (Figure 4I and data not shown), suggesting that some other signaling cell is responsible for the ref-1 expression in the anterior descendants, or that the lost function in ABalap may be compensated by other ligand-expressing cells. We finally ablated the two daughters of ABalap (i.e., ABalapa and ABalapp). The former was proposed to be the signaling cell for ABlpaaa (Moskowitz and Rothman 1996), whereas our modeling and membrane labeling data supported a more important role for the latter in signaling ABplaaa (Figure 4, A-E). Unexpectedly, we observed that the ref- 1 expression in ABplaaa descendants after either ablation was comparable to that of wild-type worms (Figure 4J, data not shown, Figure S4, Figure S5, C-E, and Figure S6). Taken together, our results suggest that the induction of left-head specification is achieved by a multiple round of signaling from consecutive cell cycles, which is especially true during the late stage of embryogenesis. The results also suggest redundant features of Notch signaling in regulating fate specification.

\section{Identification of the proposed cell pairs for the fourth Notch signaling in C. elegans embryo}

Previous studies suggested that one or both of the MSap daughters are the signaling cell(s) for fate specification of ABplpapp, the great-grandparent of the excretory cell (a functional equivalent of the human kidney), but the exact identities of the signaling cells remain elusive (Moskowitz and Rothman 1996; Priess 2005). To establish the identity of the signaling cell, we first examined our modeling results on cell contact, which suggest that only one of the MSap daughters (i.e., MSapp but not MSapa) is in contact with the excretory cell precursor (Figure 5 and Table S1). Consistent with this, a 3D projection of labeled cell membranes showed that it is MSapp but not MSapa that is in contact with the ABplpapp cell (Figure 5, A-D and Movie S2). To further validate the interaction between the two cells, we examined the lineal expression of both Notch ligands and receptors. We observed that one Notch receptor, lin-12, was expressed in all descendants of ABplp, the great-grandparent of ABplpapp (Figure 3D). Consistent with our modeling results, the GFP reporter of one Notch ligand, lag-2, was specifically expressed in MSapp but not in MSapa (Figure 5E and Figure S3, D, E, I, and J), further supporting the idea that MSapp is the signaling cell for ABplpapp. Notably, one daughter of MSapp, MSappa, was also in contact with ABplpapp, indicating that the signaling interaction is further relayed in the next cell cycle.

\section{Evidence of Notch signaling in later $A B$ descendants}

The transcription factor pal-1 is expressed in ABplppppp, the grandparent of the anal depressor muscle and an intestinal 

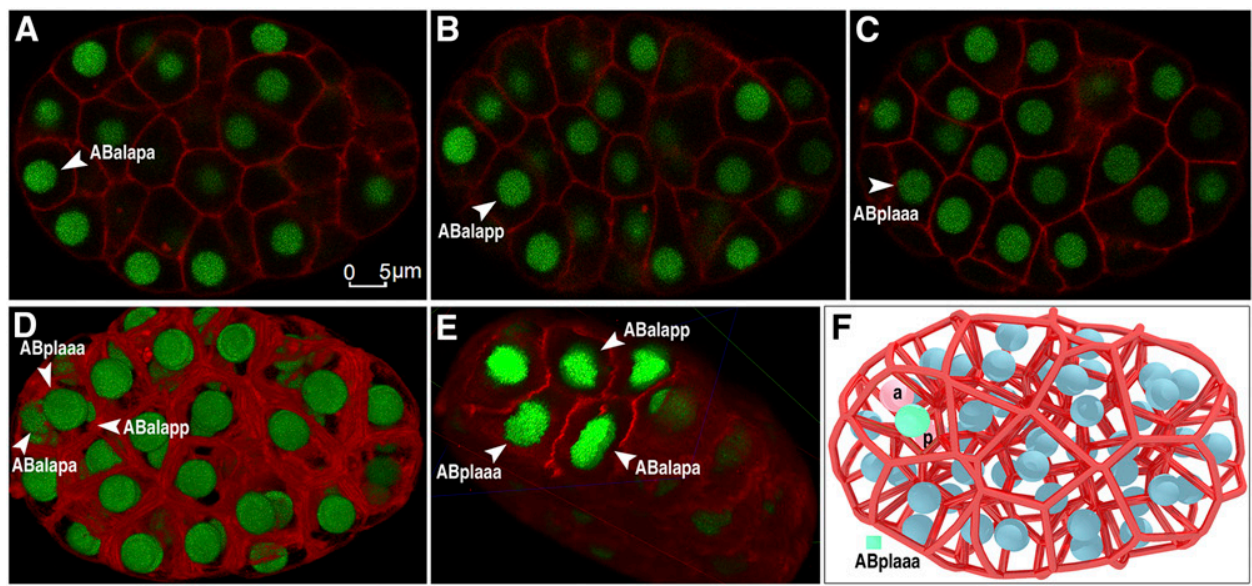

G

ABp

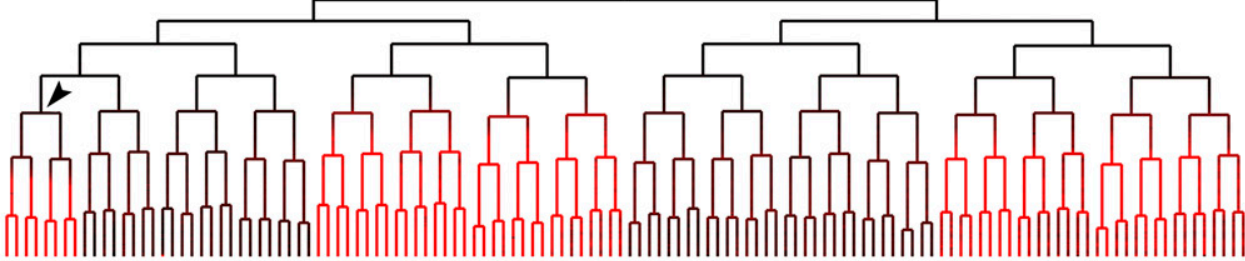

H

ABp (ABala)

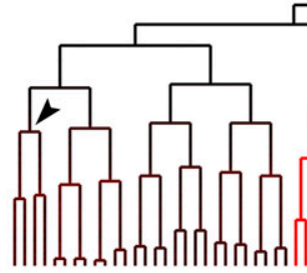

$+$
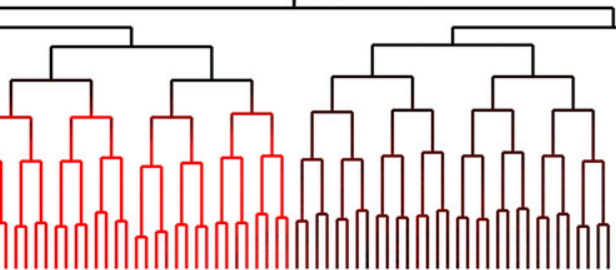

ABp (ABalap)

I

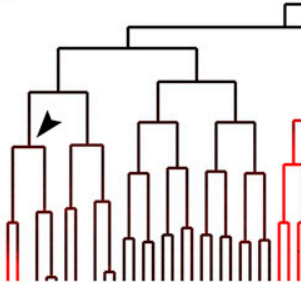

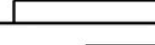

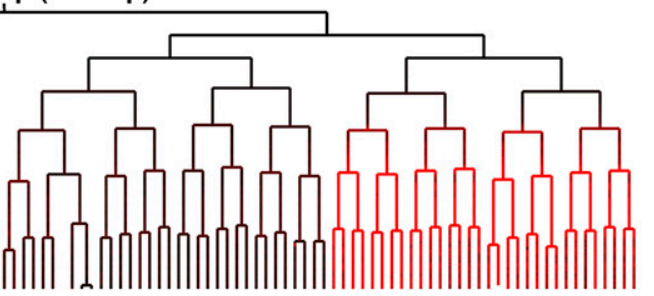

ABp (ABalapa)

J

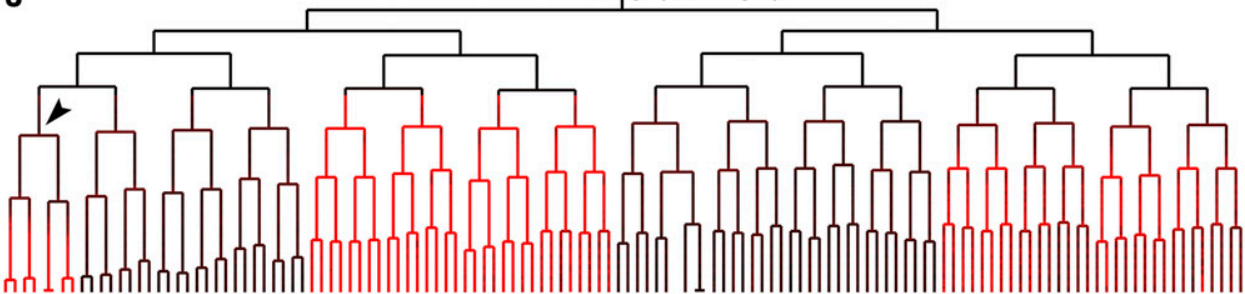

Figure 4 Refinement of third Notch signaling interaction in a 55-cell $C$. elegans embryo. (A-C) Epifluorescence micrographs of different focal planes of the same C. elegans embryo (dorsal view with anterior to the left) focusing on cells ABplaaa (plane 26), ABalapa (plane 51) and ABalapp (plane 72), respectively, as indicated by arrowheads. Cell membranes and nuclei are colored in red and green, respectively. (D) Threedimensional (3D) projection of epifluorescence micrographs. (E) Cut-open view of the 3D projection in (D) showing cell boundaries. The projection is orientated to facilitate visualization of the cell boundaries. (F) Modeling of cell boundaries in an embryo at approximately the same stage as in (D). Nuclei of ABalapa and ABalapp are colored in red and indicated with an "a" and " $p$," respectively, and the remaining nuclei colored in blue; ABplaaa nucleus is colored in green. (G-J) Lineal expression of Pref$1:: m$ Cherry in the "ABp" lineage of a wild-type embryo (G) or embryos with cell ablation (ablated cell indicated in parenthesis). Target cells of the third Notch signaling interaction are indicated with an arrowhead. muscle, and appears to be a direct target of Notch signaling required for rectal development (Edgar et al. 2001). The signaling cells for this interaction appear to be descendants of MSapa or MSapp (Priess 2005), but the exact identities of the signaling cells remain elusive. Our modeling results predicted a reproducible cell contact between MSappp and ABplpppp, the parent of ABplppppp (Figure 6D and Table S1). Cell membrane labeling and a space-filling model support the contact between the two cells (Figure 6, A-D), but not between MSapa daughters and ABplpppp (Table S1), demonstrating that MSappp is more likely to be the signaling cell for ABplpppp that is required for pal-1 expression in ABplppppp.

In a wild-type embryo of approximately the 300-cell stage, a contact between two bilaterally symmetric $\mathrm{AB}$ descendants, ABplpapppp and ABprpapppp, appears to be required for a Notch interaction for the former to develop into a neuron and a rectal epithelial cell (Bowerman et al. 1992). Our modeling 

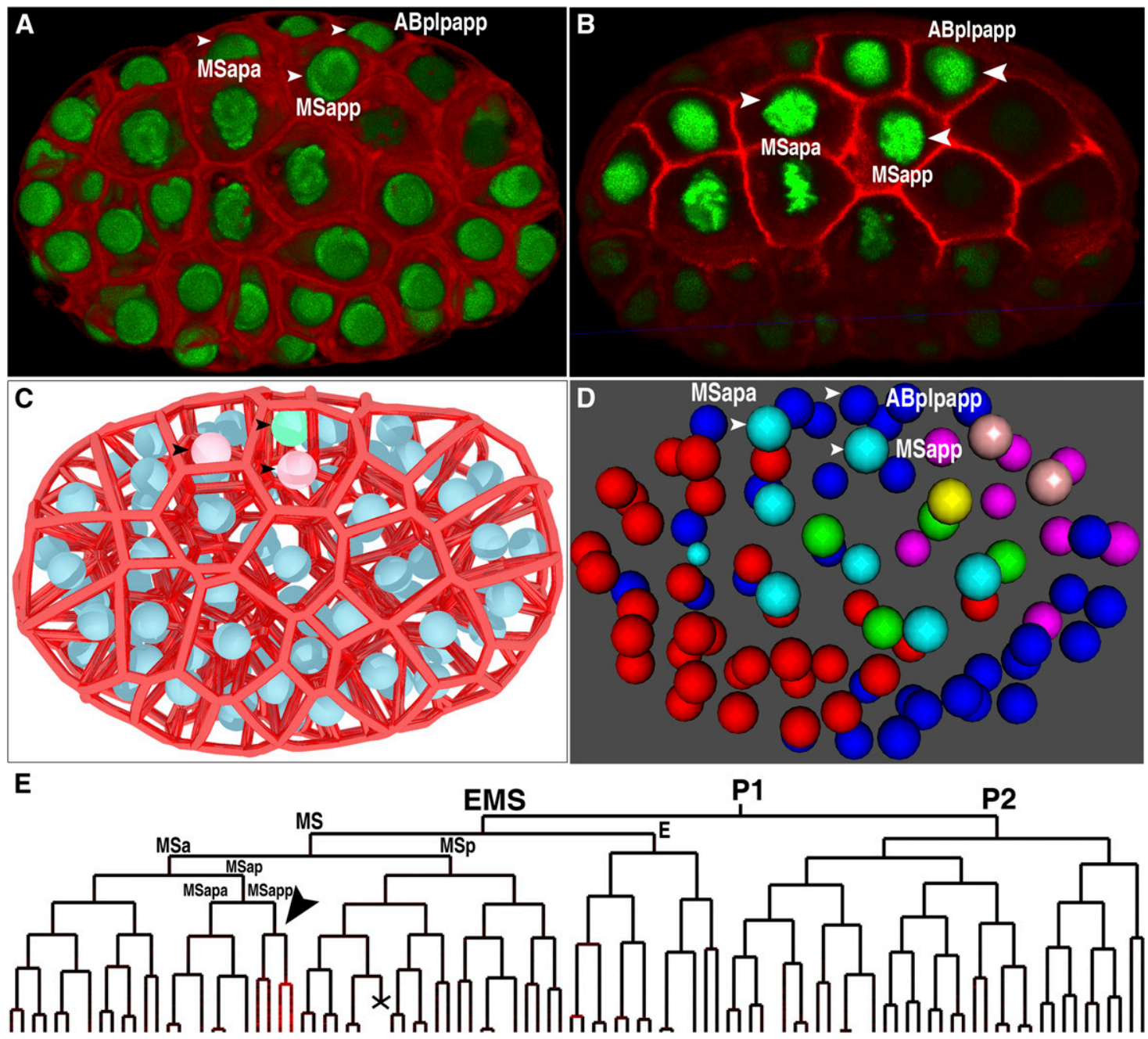

Figure 5 Refined fourth Notch signaling interaction in a C. elegans embryo. (A) Shown is a three-dimensional (3D) projection of an epifluorescence micrograph of an 87-cell embryo with cell membranes labeled by mCherry (red) and nuclei by GFP. One or both of the MSap daughters were previously proposed to signal excretory cell precursor, ABplpapp. The three cells are indicated with an arrowhead. (B) Cut-open view of the same embryo as in (A) showing cell boundaries. The embryo is oriented so that the boundaries of interest are most obvious. (C) Modeling of cell boundaries of the same embryo as in (B) with the same three cells indicated with an arrowhead. ABplpapp is colored in green, the two MSap daughters in red, and the remaining nuclei in blue. (D) 3D space-filling model of an embryo at the same stage as in (A). Red, ABa; dark blue, ABp; light blue, MS; green, E; pink, C; brown, D; and yellow, P4. The same three cells as in (A) are indicated with an arrowhead. (E) Lineal expression of a Notch ligand, lag-2, in MSapp (red) indicated with an arrowhead. Cell death is indicated with an "X."

results predicted a contact between the two cells with a high level of confidence (Table S1). Lineal expression of a Notch receptor, lin-12, was observed in ABplpapppp (Figure 3D), although the expression of both of our Notch ligands was not observed in ABprpapppp, suggesting that other Notch ligands may be involved in the interaction.

\section{A web-based utility for access to the cell-cell contact data over C. elegans embryogenesis}

To facilitate the intuitive use of our cell contact map, we developed a webpage that allows online query and navigation of cell contacts over embryogenesis (Figure S7). One can access the contacts relevant to the cell of interest by searching for the cell name or by navigating through a lineage tree. The output will show all cells that are in contact with the cell of interest in a graphical representation in which the thickness of the bars is proportional to the predicted score of a specific contact. The website is accessible through the link: http:// cccom.bionetworks.ml/.

\section{Discussion}

Signaling interaction plays a key role in the breaking of division symmetry during metazoan development. Accurate and systematic identification of the interactions at cellular resolution during development is critical for understanding the molecular mechanism of symmetry breaking but is technically challenging (Zacharias et al. 2015). This is especially true during a late proliferative stage of embryogenesis due to the difficulties in establishing contacting cells and 

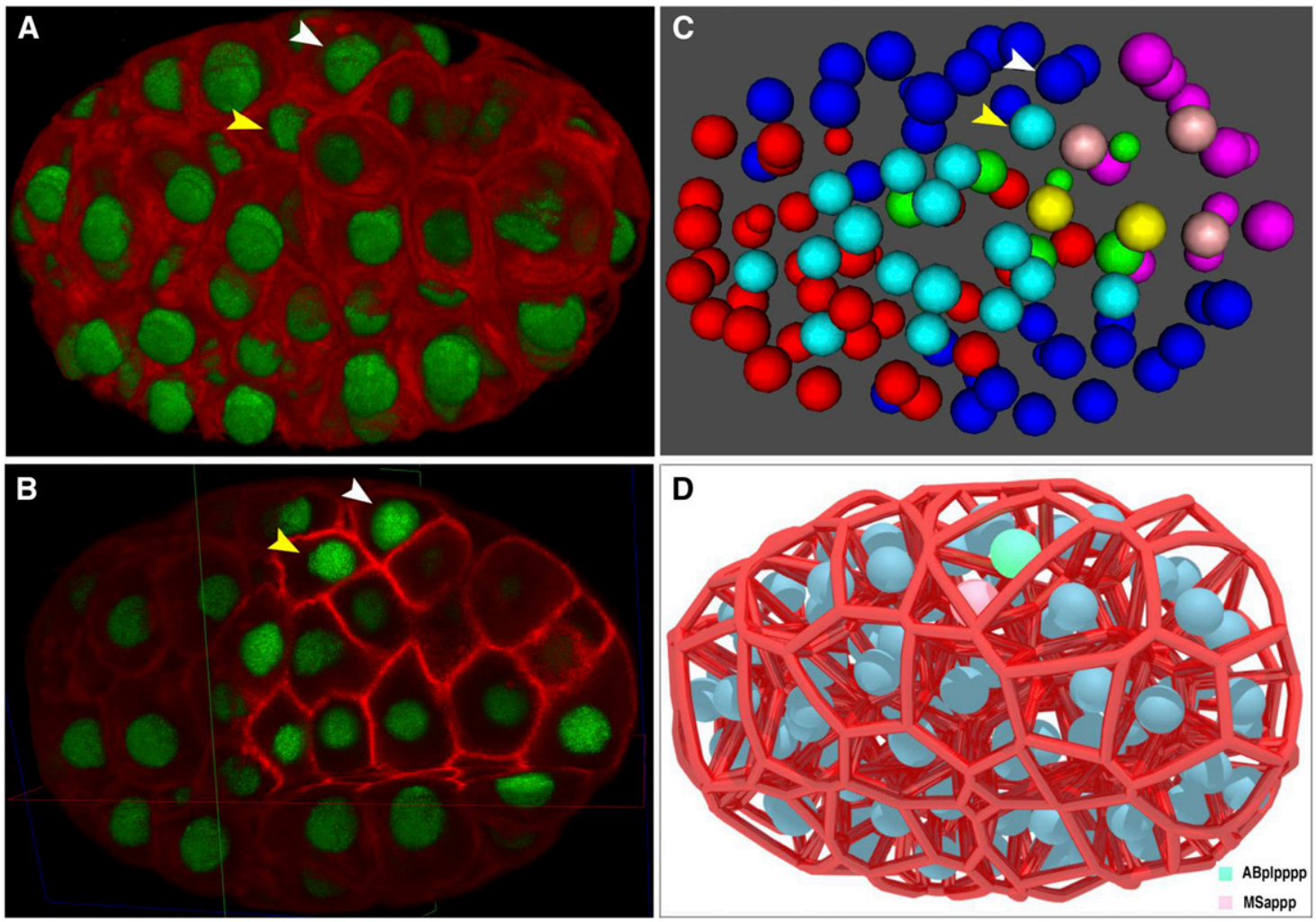

Figure 6 Identities of cells for fifth Notch signaling interaction in a C. elegans embryo. (A) Three-dimensional (3D) projection of a 96-cell embryo with cell membranes and nuclei colored in red and green, respectively. Signaling interaction was proposed to take place between two cells, MSappp (yellow arrowhead) and ABplpppp (white arrow head). (B) Cut-open view of the same embryo as in (A) showing cell boundaries. (C) 3D space-filling model of a 96-cell C. elegans embryo with cell pairs similarly color coded as in (A). (D) Modeling of cell boundaries in a 96-cell C. elegans embryo. Nuclei of MSappp and $\mathrm{ABplpppp}$ are colored in red and green, respectively, and the remaining nuclei in blue.

their identities (Bao et al. 2006; Richards et al. 2013). It is also challenging to generate the native expression dynamics of signaling molecules at cellular resolution for each cell cycle.

Here, we present an automated platform that allows the accurate identification of signaling interactions at cellular resolution during the proliferative stage of $C$. elegans embryogenesis. This was achieved by a combination of computer modeling of cell contacts, automated cell lineaging, and single-cell gene expression profiling. The cell contact map calibrated with both membrane labeling and known signaling interactions lays a foundation for the systematic identification of signaling interactions. Applying the platform in C. elegans not only allows validation and refinement of the existing Notch signaling interactions but also permits the identification of multiple novel signaling interactions, especially during a relatively late embryonic stage. The method can be applied to the characterization of any other signaling interactions. It should be noted that Voronoi modeling is an approximation of the cell surface. Predicted interactions with a smaller surface area in contact may or may not be functionally relevant. Alternatively, some functional contacts might be missed out in our list due the empirical cutoff we used in the modeling process. Functional testing is required for functional calling of a functional contact to be made.
Although many existing fate specifications have been proposed to be triggered by a single signaling event, our analyses suggest that fate specification may depend on multiple signaling interactions that take place consecutively across cell divisions. For example, though our cell contact data and membrane labeling results support that it is ABalapa that mediates the third Notch interaction (Figure 4) instead of ABalapp, as described previously (Moskowitz and Rothman 1996), ablation of either ABalapa or ABalapp does not affect ref-1 expression in ABplaaa descendants. We propose that the relay of signaling interactions over multiple generations may be a common practice for the breaking of division symmetry, as suggested earlier based on the lineal expression of Wnt components (Zacharias et al. 2015). Alternatively, the interaction might be very brief. Once the signaling cell is born, the signaling event might happen very quickly and ablation of that cell soon after its birth might not be enough to block the signaling interaction. We also observed frequent redundancy of signaling interactions that may serve to increase the robustness of a developmental process.

All of the expression patterns for Notch ligands and receptors are derived from a fusion between their promoter sequences and GFP with a heterogeneous 3'-UTR from his-72. Therefore, these vectors may capture only the zygotic but not maternal expression (Murray et al. 2008). In addition, 
the arbitrarily chosen fragment may not necessarily contain all of the functional elements required to drive its native expression. Because all of the expression patterns are derived from a single-copy transgene, some of them may be too dim to be detectable. Therefore, certain expressing cells or stages may be missing in our data set. For example, the expression of lag-2 was seen in ABala descendants by extrachromosomal array (Moskowitz and Rothman 1996), but not in our transgenic strain (Figure S3, D and E), which could be because the expression driven by a single-copy transgene is too dim to be detected or because some cis-elements are lacking in the promoter used. Use of a brighter reporter, e.g., Ruby3 (Bajar et al. 2016), may facilitate the visualization of single-copy transgenes. In summary, we present a new map of cell-cell contacts in C. elegans embryogenesis. We applied the map together with 4D imagingbased cell lineage analysis to refine previously described cell inductions. Finally, we have developed a website that has the potential to become a valuable resource for the $C$. elegans community for intuitive and easy access to cell-cell contacts.

\section{Acknowledgments}

We thank W. S. Chung for logistic support and helpful discussion with the members of Z. Zhao's laboratory. This work is supported by the Hong Kong Research Grants Council (project numbers HKBU5/CRF/11G, HKBU263512, and HKBU12103314), a Hong Kong Baptist University (HKBU) Faculty Research Fund (FRG2/13-14/063), the HKBU Strategic Development Fund for Environmental Genetics, the National Natural Science Foundation of China (project 61702396), and the China Postdoctoral Science Foundation (Project 2016M600769). Some of the strains used in this study were provided by the Caenorhabditis Genetics Center, which is funded by National Institutes of Health Office of Research Infrastructure Programs (P40 OD-010440).

Author contributions: L.C. modeled the cell contacts and H.C.K.N. contributed to the data set. M.-K.W. generated 3D projections of membrane labeling and performed cell ablation. V.W.S.H., L.-Y.C., and X.R. made the transgenic strains and produced lineal expressions. H.Y. and Z.Z. conceived the project. L.C. and Z.Z. wrote the manuscript.

\section{Literature Cited}

Aurenhammer, F., 1991 Voronoi diagrams-a survey of a fundamental geometric data structure. ACM Comput. Surv. 23: 345405. https://doi.org/10.1145/116873.116880

Bajar, B. T., E. S. Wang, A. J. Lam, B. B. Kim, C. L. Jacobs et al., 2016 Improving brightness and photostability of green and red fluorescent proteins for live cell imaging and FRET reporting. Sci. Rep. 6: 20889. https://doi.org/10.1038/srep20889

Bao, Z., J. I. Murray, T. Boyle, S. L. Ooi, M. J. Sandel et al., 2006 Automated cell lineage tracing in Caenorhabditis elegans. Proc. Natl. Acad. Sci. USA 103: 2707-2712. https://doi. org/10.1073/pnas.0511111103

Bowerman, B., F. E. Tax, J. H. Thomas, and J. R. Priess, 1992 Cell interactions involved in development of the bilaterally symmetrical intestinal valve cells during embryogenesis in Caenorhabditis elegans. Development 116: 1113-1122.

Clevers, H., and R. Nusse, 2012 Wnt/ $\beta$-Catenin signaling and disease. Cell 149: 1192-1205. https://doi.org/10.1016/j.cell.2012.05.012

Du, Z., A. Santella, F. He, M. Tiongson, and Z. Bao, 2014 De novo inference of systems-level mechanistic models of development from live-imaging-based phenotype analysis. Cell 156: 359-372. https://doi.org/10.1016/j.cell.2013.11.046

Edgar, L. G., S. Carr, H. Wang, and W. B. Wood, 2001 Zygotic expression of the caudal Homolog pal-1 is required for posterior patterning in Caenorhabditis elegans embryogenesis. Dev. Biol. 229: 71-88. https://doi.org/10.1006/dbio.2000.9977

Frøkjær-Jensen, C., M. W. Davis, M. Sarov, J. Taylor, S. Flibotte et al., 2014 Random and targeted transgene insertion in Caenorhabditis elegans using a modified Mos1 transposon. Nat. Methods 11: 529-534. https://doi.org/10.1038/nmeth.2889

Goldstein, B., 1992 Induction of gut in Caenorhabditis elegans embryos. Nature 357: 255-257. https://doi.org/10.1038/357255a0

Good, K., R. Ciosk, J. Nance, A. Neves, R. J. Hill et al., 2004 The T-box transcription factors TBX-37 and TBX-38 link GLP-1/Notch signaling to mesoderm induction in C. elegans embryos. Development 131: 1967-1978. https://doi.org/10.1242/dev.01088

Greenwald, I., 2013 Notch signaling: genetics and structure (January 17, 2013), WormBook, ed. The C. elegans Research Community, WormBook, doi/10.1895/wormbook.1.10.2, http://www. wormbook.org. https://doi.org/10.1895/wormbook.1.10.2

Greenan, G., C. P. Brangwynne, S. Jaensch, J. Gharakhani, F. Julicher et al., 2010 Centrosome size sets mitotic spindle length in Caenorhabditis elegans embryos. Curr. Biol. 20: 353-358. https://doi.org/10.1016/j.cub.2009.12.050

Hara, Y., and A. Kimura, 2009 Cell-size-dependent spindle elongation in the Caenorhabditis elegans early embryo. Curr. Biol. 19: 1549-1554. https://doi.org/10.1016/j.cub.2009.07.050

Hench, J., J. Henriksson, M. Luppert, and T. R. Burglin, 2009 Spatio-temporal reference model of Caenorhabditis elegans embryogenesis with cell contact maps. Dev. Biol. 333: 113. https://doi.org/10.1016/j.ydbio.2009.06.014

Ho, V. W., M. K. Wong, X. An, D. Guan, J. Shao et al., 2015 Systemslevel quantification of division timing reveals a common genetic architecture controlling asynchrony and fate asymmetry. Mol. Syst. Biol. 11: 814. https://doi.org/10.15252/msb.20145857

Huang, S., P. Shetty, S. M. Robertson, and R. Lin, 2007 Binary cell fate specification during C. elegans embryogenesis driven by reiterated reciprocal asymmetry of TCF POP-1 and its coactivator -catenin SYS-1. Development 134: 2685-2695. https://doi. org/10.1242/dev.008268

Hutter, H., and R. Schnabel, 1994 glp-1 and inductions establishing embryonic axes in C. elegans. Development 120: 2051-2064.

Hutter, H., and R. Schnabel, 1995 Specification of anterior-posterior differences within the $\mathrm{AB}$ lineage in the C. elegans embryo: a polarising induction. Development 121: 1559-1568.

Keller, P. J., A. D. Schmidt, J. Wittbrodt, and E. H. Stelzer, 2008 Reconstruction of zebrafish early embryonic development by scanned light sheet microscopy. Science 322: 10651069. https://doi.org/10.1126/science.1162493

Mello, C. C., B. W. Draper, and J. R. Priess, 1994 The maternal genes apx-1 and glp-1 and establishment of dorsal-ventral polarity in the early C. elegans embryo. Cell 77: 95-106. https:// doi.org/10.1016/0092-8674(94)90238-0

Mickey, K. M., C. C. Mello, M. K. Montgomery, A. Fire, and J. R. Priess, 1996 An inductive interaction in 4-cell stage C. elegans embryos involves APX-1 expression in the signalling cell. Development 122: 1791-1798.

Moore, J. L., Z. Du, and Z. Bao, 2013 Systematic quantification of developmental phenotypes at single-cell resolution during embryogenesis. Development 140: 3266-3274. https://doi.org/ 10.1242/dev.096040 
Moskowitz, I. P., and J. H. Rothman, 1996 lin-12 and glp-1 are required zygotically for early embryonic cellular interactions and are regulated by maternal GLP-1 signaling in Caenorhabditis elegans. Development 122: 4105-4117.

Murray, J. I., Z. Bao, T. J. Boyle, M. E. Boeck, B. L. Mericle et al., 2008 Automated analysis of embryonic gene expression with cellular resolution in C. elegans. Nat. Methods 5: 703-709. https://doi.org/10.1038/nmeth.1228

Murray, J. I., T. J. Boyle, E. Preston, D. Vafeados, B. Mericle et al., 2012 Multidimensional regulation of gene expression in the C. elegans embryo. Genome Res. 22: 1282-1294. https://doi. org/10.1101/gr.131920.111

Muzzey, D., and A. van Oudenaarden, 2009 Quantitative timelapse fluorescence microscopy in single cells. Annu. Rev. Cell Dev. Biol. 25: 301-327. https://doi.org/10.1146/annurev.cellbio.042308.113408

Neves, A., and J. R. Priess, 2005 The REF-1 family of bHLH transcription factors pattern C. elegans embryos through Notch-dependent and Notch-independent pathways. Dev. Cell 8: 867-879. https:// doi.org/10.1016/j.devcel.2005.03.012

Okabe, A., B. Boots, K. Sugihara, and S. N. Chiu, 2000 Spatial Tessellations: Concepts and Applications of Voronoi Diagrams. John Wiley \& Sons, New York.

Priess, J. R., 2005 Notch signaling in the C. elegans embryo (June $25,2005)$ WormBook, ed. The C. elegans Research Community, WormBook, doi/10.1895/wormbook.1.4.1, http://www.wormbook.org.

Richards, J. L., A. L. Zacharias, T. Walton, J. T. Burdick, and J. I. Murray, 2013 A quantitative model of normal Caenorhabditis elegans embryogenesis and its disruption after stress. Dev. Biol. 374: 12-23. https://doi.org/10.1016/j.ydbio.2012.11.034

Rocheleau, C. E., W. D. Downs, R. Lin, C. Wittmann, Y. Bei et al., 1997 Wnt signaling and an APC-related gene specify endoderm in early C. elegans embryos. Cell 90: 707-716. https:// doi.org/10.1016/S0092-8674(00)80531-0

Rose, L., and P. Gonczy, 2014 Polarity establishment, asymmetric division and segregation of fate determinants in early C. elegans embryos (December 30, 2014), WormBook, ed. The C. elegans Research Community, WormBook, doi/10.1895/wormbook.1.30.2, http://www.wormbook.org.

Rycroft, C. H., 2009 VORO++ : a three-dimensional Voronoi cell library in $\mathrm{C}++$. Chaos 19: 041111.

Sawa, H., 2012 Control of cell polarity and asymmetric division in C. elegans. Curr. Top. Dev. Biol. 101: 55-76.

Schnabel, R., H. Hutter, D. Moerman, and H. Schnabel, 1997 Assessing normal embryogenesis in Caenorhabditis elegans using a 4D microscope: variability of development and re- gional specification. Dev. Biol. 184: 234-265. https://doi.org/ 10.1006/dbio.1997.8509

Schnabel, R., M. Bischoff, A. Hintze, A.-K. Schulz, A. Hejnol et al., 2006 Global cell sorting in the C. elegans embryo defines a new mechanism for pattern formation. Dev. Biol. 294: 418-431. https://doi.org/10.1016/j.ydbio.2006.03.004

Shah, P. K., A. Santella, A. Jacobo, K. Siletti, A. J. Hudspeth et al., 2017 An in toto approach to dissecting cellular interactions in complex tissues. Dev. Cell 43: 530-540.e4. https://doi.org/ 10.1016/j.devcel.2017.10.021

Shao, J., K. He, H. Wang, W. S. Ho, X. Ren et al., 2013 Collaborative regulation of development but independent control of metabolism by two epidermis-specific transcription factors in Caenorhabditis elegans. J. Biol. Chem. 288: 33411-33426. https:// doi.org/10.1074/jbc.M113.487975

Shelton, C. A., and B. Bowerman, 1996 Time-dependent responses to glp-1-mediated inductions in early C. elegans embryos. Development 122: 2043-2050.

Sulston, J. E., E. Schierenberg, J. G. White, and J. N. Thomson, 1983 The embryonic cell lineage of the nematode Caenorhabditis elegans. Dev. Biol. 100: 64-119. https://doi.org/10.1016/ 0012-1606(83)90201-4

Yochem, J., K. Weston, and I. Greenwald, 1988 The Caenorhabditis elegans lin-12 gene encodes a transmembrane protein with overall similarity to Drosophila Notch. Nature 335: 547-550. https://doi.org/10.1038/335547a0

Zacharias, A. L., T. Walton, E. Preston, and J. I. Murray, 2015 Quantitative differences in nuclear $\beta$-catenin and TCF pattern embryonic cells in C. elegans. PLoS Genet 11: e1005585.

Zacharias, A. L., and J. I. Murray, 2016 Combinatorial decoding of the invariant C. elegans embryonic lineage in space and time. Genesis 54: 182-197. https://doi.org/10.1002/dvg.22928

Zhao, Z., T. J. Boyle, Z. Bao, J. I. Murray, B. Mericle et al., 2008 Comparative analysis of embryonic cell lineage between Caenorhabditis briggsae and Caenorhabditis elegans. Dev. Biol. 314: 93-99. https://doi.org/10.1016/j.ydbio.2007.11.015

Zhao, Z., S. Flibotte, J. I. Murray, D. Blick, T. J. Boyle et al., 2010a New tools for investigating the comparative biology of Caenorhabditis briggsae and C. elegans. Genetics 184: 853-863. https://doi.org/10.1534/genetics.109.110270

Zhao, Z., T. J. Boyle, Z. Liu, J. I. Murray, W. B. Wood et al., 2010b A negative regulatory loop between microRNA and Hox gene controls posterior identities in Caenorhabditis elegans. PLoS Genet. 6: e1001089. https://doi.org/10.1371/journal.pgen.1001089

Communicating editor: O. Hobert 